\title{
强酸性阳离子交换树脂催化水相法合成双吲哚甲烷类衍生物
}

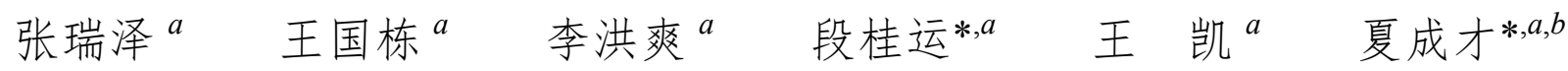 \\ ( ${ }^{a}$ 山东第一医科大学(山东省医学科学院)药学院 泰安 271016) \\ $\left({ }^{b}\right.$ 山东第一医科大学(山东省医学科学院)药理研究所 泰安 271016)
}

\begin{abstract}
摘要 开发了一种通过强酸性离子交换树脂催化将苯甲醛和吲哚转化成双吲哚甲烷类衍生物的有效合成方法, 它是以 水作为溶剂, 实现了催化剂可循环使用 6 次, 以较高收率得到了多种双吲哚甲烷化合物.

关键词＼cjkstart强酸性阳离子交换树脂; 吲哚; 双吲哚甲烷; 缩合
\end{abstract}

\section{Strong-Acid Cation Exchange Resin Catalyzed Synthesis of Bis(indolyl)methanes in Water}

\author{
Zhang, Ruize ${ }^{a}$ \\ Wang, Guodong ${ }^{a}$ \\ Li, Hongshuang ${ }^{a}$ \\ Duan, Guiyun*,a \\ Wang, Kai ${ }^{a}$ \\ Xia, Chengcai ${ }^{*, a, b}$ \\ ( ${ }^{a}$ Pharmacy College, Shandong First Medical University \& Shandong Academy of Medical Sciences, Tai'an 271016) \\ ( ${ }^{b}$ Institute of Pharmacology, Shandong First Medical University \& Shandong Academy of Medical Sciences, Tai'an 271016)
}

\begin{abstract}
An efficient protocol for conversion of aldehyde and indole into bis(indolyl)methanes derivatives catalyzed by strong-acid cation exchange resin has been developed. The $\mathrm{H}_{2} \mathrm{O}$ was used as solution and recycle catalyst can be used six times. Various bis(indolyl)methanes derivatives were obtained in excellent yields.

Keywords strong-acid cation exchange resin; indole; bis(indolyl)methanes; condensation
\end{abstract}

\section{Introduction}

The bis(indolyl)methanes (BIMs) have been widely found in various terrestrial and marine natural resources. ${ }^{[1]}$ These compounds present various important biological and pharmacological activities, ${ }^{[2]}$ such as antileishmanial, ${ }^{[3]}$ antibacterial, anti-inflammatory, ${ }^{[4]}$ antihyperlipidemic, ${ }^{[5]}$ anxiolytic ${ }^{[6]}$ and anticance. ${ }^{[7]}$ They also are used as intermediates in agrochemicals, ${ }^{[8]}$ electrochemical properties ${ }^{[9]}$ and material science. ${ }^{[10]}$ Due to the important pharmacological properties of BIMs, various methods have been reported for the synthesis of these derivatives in the literature.

Bisindolylmethanes have been synthesized in moderate to very good yields based on the reaction of indoles with aldehydes in the presence a wide range of catalysts. The most of these catalysts reported in the literature are Lewis acids, such as $\mathrm{FeSO}_{4},{ }^{[11]} \mathrm{CdS}^{[12]}$ sulfated anatase titania $\left(\mathrm{TiO}_{2}-\mathrm{SO}_{4}{ }^{2-}\right),{ }^{[13]}$ oxalic acid, ${ }^{[14]}$ succinimide- $N$-sulfonic acid, ${ }^{[15]}$ triethyl benzylammonium chloride ${ }^{[16]}$ trichloroi- socyanuric acid, ${ }^{[17]} \mathrm{CeCl}_{3},{ }^{[18]} \mathrm{Br}_{2}{ }^{[19]}$ and sulfated polyborate. ${ }^{[20]}$ And ion exchange resin, ${ }^{[21]}$ montmorillonite, ${ }^{[22]}$ tetranuclear $\mathrm{Zn} / 4 \mathrm{f}$ coordination clusters, ${ }^{[23]}$ magnetic solid acid nano-catalysts, ${ }^{[24]}$ nano-catalyst, ${ }^{[25]}$ polyacrylonitrile fiber, ${ }^{[26]}$ bakers' yeast ${ }^{[27]}$ all can catalyze this reaction. Also, the condensation occurs without the addition of catalysts under ultrasound irradiation ${ }^{[28]}$ or high temperature. ${ }^{[29]}$ The ion exchange resins, such as Amberlyst-15, Amberlyst-35 and Indion Ina $225 \mathrm{H}$, with high specific surface area were found the most effective for reactions of indoles with aldehydes to afford the corresponding bis(indolyl)methanes in excellent yields. ${ }^{[21]}$ It is a heterogeneous, selective, and eco-benign, and can be recovered and reused for several runs without loss of activity. They all react in an organic solvent such as acetonitrile, dichloroethane and acetone. As part of our ongoing interest in development of environmentally friendly synthetic methodologies ${ }^{[30]}$ and aqueous-phase synthesis technology, ${ }^{[31]}$ herein, we disclose a green and efficient synthetic strategy for preparation of bis(indolyl)methanes via the condensation of indoles with

\footnotetext{
* Corresponding author. E-mail: xiachc@163.com

Received October 11, 2018; revised December 18, 2018; published online January 18, 2019.

Project supported by the Medical and Health Technology Development Program in Shandong Province (No. 2015WS0102).

山东省医药卫生科技发展计划(No. 2015 WS0102)资助项目.
} 
aldehydes in water catalyzed by strong-acid cation exchange resin.

\section{Results and discussion}

We initiated our investigation on the model reaction of benzaldehyde (1a) with indole (2a) to optimize the reaction parameters (Table 1). To our delight, the condensation took place in the presence of Amberlite $732(10 \mathrm{~mol} \%)$ in $\mathrm{H}_{2} \mathrm{O}$ under $\mathrm{N}_{2}$ for $4 \mathrm{~h}$, the desired product was acquired in 97\% yield (Entry 2, Table 1). Thus, Amberjet IMAC HP1110, Amberlite IR-120, Amberlite XAD 7HP, Amberlyst 15 , Dowex 50WX8 and free catalyst were tested during this reaction, in which Amberlite 732 gave the best result (Entries 1 7, Table 1). Secondly, the effects of solvent were examined. Water was found to be effective solvent compared to EtOH, toluene, dichloroethane, $\mathrm{N}, \mathrm{N}$-dimethylformamide (DMF), $\mathrm{CH}_{3} \mathrm{CN}$ and dimethyl sulfoxide (DMSO) (Entries 8 13, Table 1). When the temperature was reduced to room temperature or increased to $120{ }^{\circ} \mathrm{C}$, the yield decreased to $23 \%$ and $94 \%$ (Entries $14 \sim 18$, Table 1). Finally, when the reaction times were shortened or delayed, the yield of $\mathbf{2 a}$ decreased (Entries 1 and 19 23, Table 1). From the results shown above, the optimized condensation conditions were identified as follows: $1 \mathrm{a}(0.5 \mathrm{mmol})$, indole $(1.0 \mathrm{mmol}), 10 \mathrm{~mol} \%$ of amberlite 732 as catalyst, and $\mathrm{H}_{2} \mathrm{O}(2.0 \mathrm{~mL})$ as solvent, at $100{ }^{\circ} \mathrm{C}$ under an $\mathrm{N}_{2}$ atmosphere for $4 \mathrm{~h}$.

The representative results were summarized in Table 2, which the condensation of various aldehydes with indoles was obtained under the influence of the amberlite 732 in $\mathrm{H}_{2} \mathrm{O}$ at $100{ }^{\circ} \mathrm{C}$ for $4 \mathrm{~h}$. A series of aldehydes were allowed to react with indoles, affording bis(indolyl)methanes in moderate to good yields. Generally, no remarkable differences in reactivity were observed when electron-donor groups or electron-withdrawing were present in the aromatic ring $(\mathbf{3 a} \sim \mathbf{3 j})$. The reaction has slight impact on

Table 1 Optimization of reaction conditions ${ }^{a}$

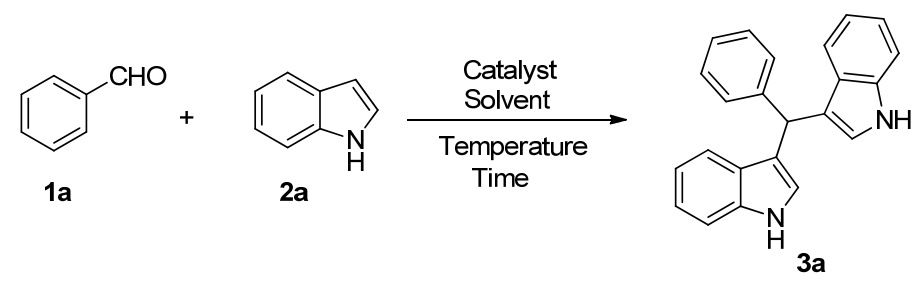

\begin{tabular}{|c|c|c|c|c|c|}
\hline Entry & Catalyst & Solvent & Temperature $/{ }^{\circ} \mathrm{C}$ & Time/h & Yield $^{b} / \%$ \\
\hline 1 & - & $\mathrm{H}_{2} \mathrm{O}$ & 100 & 4 & 82 \\
\hline 2 & Amberlite 732 & $\mathbf{H}_{2} \mathrm{O}$ & 100 & 4 & $\mathbf{9 7}, 81^{c}$ \\
\hline 3 & Amberjet IMAC HP1110 & $\mathrm{H}_{2} \mathrm{O}$ & 100 & 4 & 93 \\
\hline 4 & Amberlite IR-120 & $\mathrm{H}_{2} \mathrm{O}$ & 100 & 4 & 91 \\
\hline 5 & Amberlite XAD 7HP & $\mathrm{H}_{2} \mathrm{O}$ & 100 & 4 & 90 \\
\hline 6 & Amberlyst 15 & $\mathrm{H}_{2} \mathrm{O}$ & 100 & 4 & 86 \\
\hline 7 & Dowex 50WX8 & $\mathrm{H}_{2} \mathrm{O}$ & 100 & 4 & 88 \\
\hline 8 & HOAc & $\mathrm{H}_{2} \mathrm{O}$ & 100 & 4 & 90 \\
\hline 9 & Methanesulfonic acid & $\mathrm{H}_{2} \mathrm{O}$ & 100 & 4 & 92 \\
\hline 10 & $p$-toluenesulfonic acid & $\mathrm{H}_{2} \mathrm{O}$ & 100 & 4 & 87 \\
\hline 11 & Amberlite 732 & EtOH & 100 & 4 & 16 \\
\hline 12 & Amberlite 732 & Toluene & 100 & 4 & 9 \\
\hline 13 & Amberlite 732 & Dichloroethane & 100 & 4 & 27 \\
\hline 14 & Amberlite 732 & DMF & 100 & 4 & 25 \\
\hline 15 & Amberlite 732 & $\mathrm{CH}_{3} \mathrm{CN}$ & 100 & 4 & 6 \\
\hline 16 & Amberlite 732 & DMSO & 100 & 4 & 37 \\
\hline 17 & Amberlite 732 & $\mathrm{H}_{2} \mathrm{O}$ & 25 & 4 & 23 \\
\hline 18 & Amberlite 732 & $\mathrm{H}_{2} \mathrm{O}$ & 40 & 4 & 25 \\
\hline 19 & Amberlite 732 & $\mathrm{H}_{2} \mathrm{O}$ & 60 & 4 & 33 \\
\hline 20 & Amberlite 732 & $\mathrm{H}_{2} \mathrm{O}$ & 80 & 4 & 65 \\
\hline 21 & Amberlite 732 & $\mathrm{H}_{2} \mathrm{O}$ & 120 & 4 & 94 \\
\hline 22 & Amberlite 732 & $\mathrm{H}_{2} \mathrm{O}$ & 100 & 0.5 & 19 \\
\hline 20 & Amberlite 732 & $\mathrm{H}_{2} \mathrm{O}$ & 100 & 1.0 & 52 \\
\hline 21 & Amberlite 732 & $\mathrm{H}_{2} \mathrm{O}$ & 100 & 2.0 & 68 \\
\hline 22 & Amberlite 732 & $\mathrm{H}_{2} \mathrm{O}$ & 100 & 6.0 & 93 \\
\hline 23 & Amberlite 732 & $\mathrm{H}_{2} \mathrm{O}$ & 100 & 8.0 & 91 \\
\hline
\end{tabular}

${ }^{a}$ Reaction conditions: 1a $(0.5 \mathrm{mmol})$, indole $(1.0 \mathrm{mmol})$, catalyst $(10 \mathrm{~mol} \%)$ and solvent $(2.0 \mathrm{~mL})$ under $\mathrm{N}_{2}$ atmosphere at $100{ }^{\circ} \mathrm{C}$ for $4 \mathrm{~h}$, unless otherwise noted. ${ }^{b}$ Isolated yields. 
Table 2 Substrate scope ${ }^{a}$

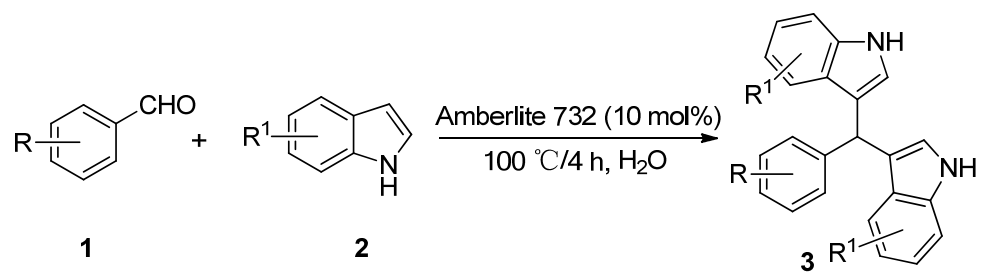

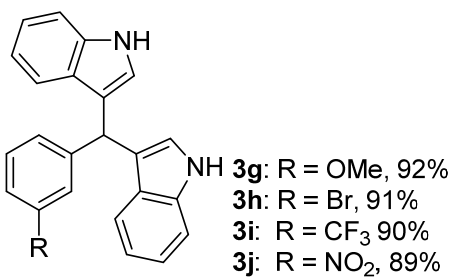<smiles>Cc1cccc(C)c1C(c1c[nH]c2ccccc12)c1c[nH]c2ccccc12</smiles>

$3 n, 77 \%$<smiles>Cc1[nH]c2ccccc2c1C(c1ccccc1)c1c(C)[nH]c2ccccc12</smiles>

$3 q, 96 \%$

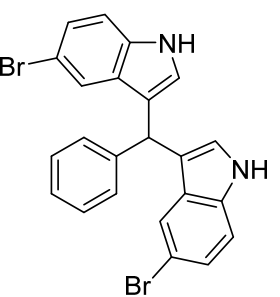

$3 r, 94 \%$<smiles>Cc1cccc2c1-c1cccc3[nH]cc(c13)C2c1c[nH]c2ccccc12</smiles>

$30,86 \%$

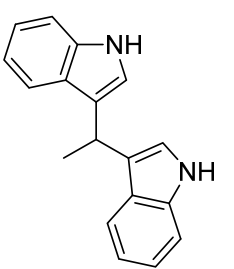

3s, $86 \%$
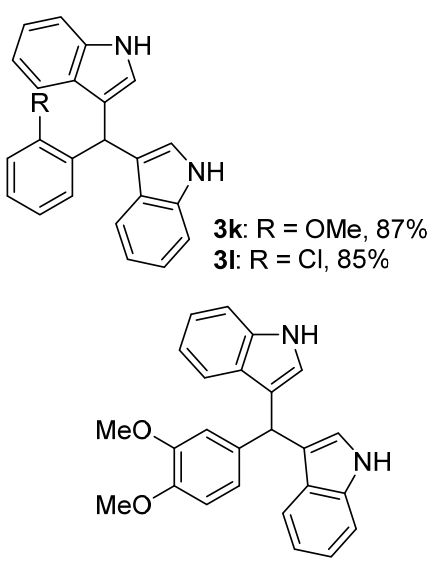

3p, $91 \%$

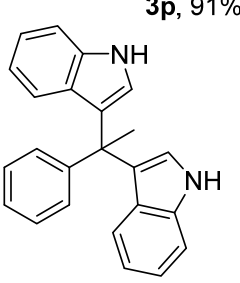

$3 t, 81 \%$

${ }^{a}$ Reaction conditions: $1(0.5 \mathrm{mmol}), 2(1.0 \mathrm{mmol})$, catalyst $(10 \mathrm{~mol} \%)$ and solvent $(2.0 \mathrm{~mL})$ under $\mathrm{N}_{2}$ atmosphere at $100{ }^{\circ} \mathrm{C}$ for $4 \mathrm{~h}$, unless otherwise noted. ${ }^{b}$ Isolated yields.

the steric hindrance of aldehydes. For example, 2-methoxybenzaldehyde and 2-chlorobenzaldehyde gave a slightly lower yield of the desired products than their $p a-$ ra-substituted homologues (3k, 31). Similarly, 2,4-dimethylbenzaldehyde, 2,3-dimethylbenzaldehyde, and 2,6-dimethylbenzaldehyde have lower yields $(\mathbf{3 m} \sim \mathbf{3 0})$ but 3,4-dimethoxybenzaldehyde has good yield (3p). We next turned our attention towards substituted indoles. 1-Methyl$1 H$-indoles and 5-bromo- $1 H$-indoles were subjected to the reaction with 1a. They all gave the corresponding DIMs 3q and $\mathbf{3 r}$ in good yields. Many aliphatic aldehydes have been tested in this reaction, such formaldehyde, acetaldehyde, propionaldehyde, but only product of acetaldehyde (3s) was obtained with $86 \%$ yield. Surprisingly, an acetophenone can react with indole (2a) to produce 3,3'-(1-phenylethane-1,1-diyl)bis( $1 H$-in- dole) (3t) in $81 \%$ yield.

The recycling of the catalytic was also investigated under the same conditions by the condensation of benzaldehyde with indoles. After completion of the reaction and adding dichloromethane $(10 \mathrm{~mL})$, the mixture was filtered and catalyst was recovered and reused (Figure 1). The re-

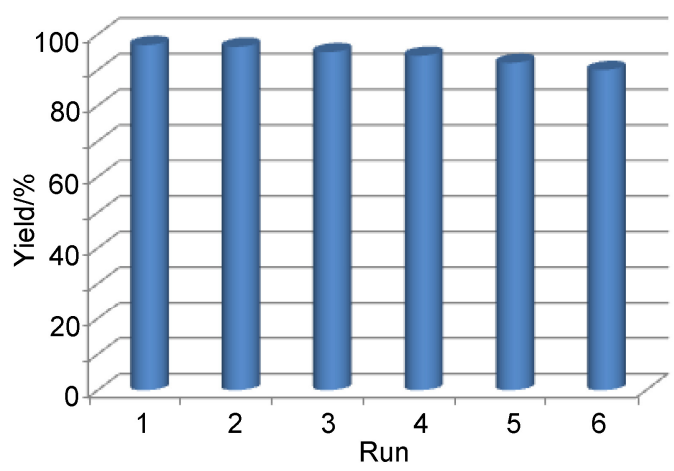

Figure 1 Recycling of the catalyst for synthesis of bis(indolyl)methanes

covered catalyst was reused six runs and resulted in a slight decrease in the catalytic activity which yield was down to $88 \%$. Pleasingly, the yield can be recovered to $94.7 \%$ after acidification of the recovered catalyst. The recovered water was reused without any loss of yield, and the color become darker than past, but the color of water 
can be bleached by activated carbon.

To demonstrate the synthetic utility of the condensation reactions, the gram-scale synthesis was carried out, affording products $\mathbf{3 a}, \mathbf{3 b}$ and $\mathbf{3 c}$ in $85 \%, 82 \%$ and $83 \%$ yields, respectively (Scheme 1). Finally, the yields of $\mathbf{3 a}$ dropped to $76 \%$ and $63 \%$ when the catalyst loading was decreased to 5 and $2.5 \mathrm{~mol} \%$ respectively in this gram-scale synthesis.

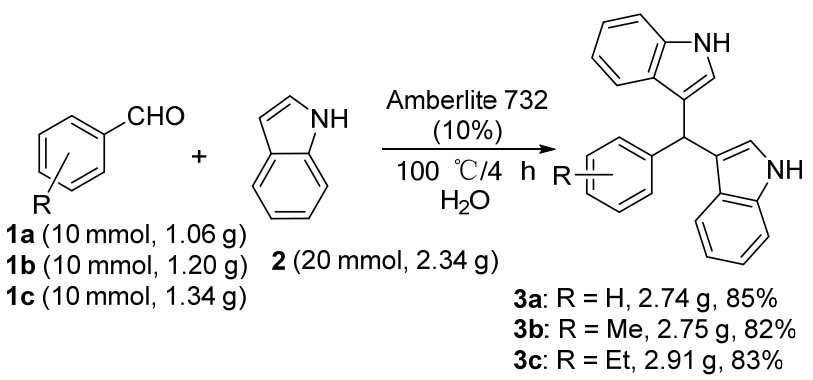

Scheme 1 Gram-scale synthesis

\section{Conclusions}

In summary, a simple method for conversion of aldehyde and indole into bis(indolyl)methanes derivative has been developed catalyzed by strong-acid cation exchange resin. The reaction proceeds under mild conditions and shows a broad substrate scope. It provides a convenient method to synthesize bis(indolyl)methanes. $\mathrm{H}_{2} \mathrm{O}$ was used as solution, and recycle catalyst can be used about six times.

\section{Experimental section}

\subsection{General information}

All reactions were run under air atmosphere in Schlenk tubes. DCE, EtOH, $\mathrm{CH}_{3} \mathrm{CN}$, toluene and DMSO were analytical grade and not distilled before use. Commercial indoles and benzaldehydes were used without purification. ${ }^{1} \mathrm{H}$ NMR and ${ }^{13} \mathrm{C}$ NMR spectra were recorded using a 400 $\mathrm{MHz}$ spectrometer in $\mathrm{CDCl}_{3}$. Melting points were determined by using a local hot-stage melting point apparatus and are uncorrected. Mass spectra were recorded using LC-MS and HRMS (ESI-TOF analyzer) equipment.

4.2 Synthesis of bis(indolyl)methanes in water catalyzed by strong-acid cation exchange resin

The amberlite 732 was wished by water for 3 times, and then soaked in 5\% hydrochloric acid about $1 \mathrm{~h}$. After this, the mixture was filtered off for separation of catalyst and wished by water again for $\mathrm{pH}$ up to 5 .

Benzaldehyde (74.3 mg, $0.7 \mathrm{mmol})$, indole $(117 \mathrm{mg}, 1.0$ $\mathrm{mmol})$, catalyst $(50 \mathrm{mg}, 0.025 \mathrm{~g} / \mathrm{mL})$ in $2 \mathrm{~mL}$ water were mixed and vigorously stirred under atmosphere at $100{ }^{\circ} \mathrm{C}$ for $4 \mathrm{~h}$. After cooling down to room temperature and adding dichloromethane $(10 \mathrm{~mL})$, the mixture was filtered off catalyst which was recovered and reused. The filtrate was extracted with dichloromethane $(10 \mathrm{~mL} \times 3)$. Subsequently, combined of organic phase and concentrated in vacuum, the residue was purified by flash chromatography on a short silica gel to afford corresponding product.

3,3'-(Phenylmethylene)bis(1H-indole) (3a): 97\% yield, reddish brown solid, m.p. 97.2 99.2 ${ }^{\circ} \mathrm{C}$; ${ }^{1} \mathrm{H}$ NMR $(400$ $\left.\mathrm{MHz}, \mathrm{CDCl}_{3}\right) \delta: 7.84(\mathrm{~s}, 2 \mathrm{H}), 7.38(\mathrm{~d}, J=7.9 \mathrm{~Hz}, 2 \mathrm{H})$, 7.33 (dd, $J=7.3,4.5 \mathrm{~Hz}, 4 \mathrm{H}), 7.27$ (t, $J=7.6 \mathrm{~Hz}, 2 \mathrm{H}), 7.21$ $(\mathrm{d}, J=7.1 \mathrm{~Hz}, 1 \mathrm{H}), 7.16(\mathrm{t}, J=7.6 \mathrm{~Hz}, 2 \mathrm{H}), 7.00(\mathrm{t}, J=7.5$ $\mathrm{Hz}, 2 \mathrm{H}), 6.62 \sim 6.61(\mathrm{~m}, 2 \mathrm{H}), 5.88(\mathrm{~s}, 1 \mathrm{H}) ;{ }^{13} \mathrm{C} \mathrm{NMR}(101$ $\left.\mathrm{MHz}, \mathrm{CDCl}_{3}\right) \delta: 143.97,136.64,128.70,128.19,127.04$, $126.11,123.57,121.89,119.91,119.66,119.19,111.01$, 40.18. HRMS $\left(\mathrm{ESI}^{+}\right.$) calcd for $\mathrm{C}_{23} \mathrm{H}_{19} \mathrm{~N}_{2}[\mathrm{M}+\mathrm{H}]$ 323.1543 , found 323.1544 .

3,3'-( $p$-Tolylmethylene)bis( $1 H$-indole) (3b): 91\% yield, reddish brown solid, m.p. $138 \sim 140{ }^{\circ} \mathrm{C} ;{ }^{1} \mathrm{H}$ NMR $(400$ $\left.\mathrm{MHz}, \mathrm{CDCl}_{3}\right) \delta: 7.82(\mathrm{~s}, 2 \mathrm{H}), 7.40(\mathrm{dd}, J=7.9 \mathrm{~Hz}, 2 \mathrm{H})$, $7.33(\mathrm{dt}, J=8.2,0.8 \mathrm{~Hz}, 2 \mathrm{H}), 7.25 \sim 7.22(\mathrm{~m}, 2 \mathrm{H}), 7.16$ (ddd, $J=8.2,7.1,1.1 \mathrm{~Hz}, 2 \mathrm{H}), 7.08$ (d, $J=7.8 \mathrm{~Hz}, 2 \mathrm{H})$, $7.00(\mathrm{ddd}, J=8.0,7.1,1.0 \mathrm{~Hz}, 2 \mathrm{H}), 6.63 \sim 6.62(\mathrm{~m}, 2 \mathrm{H})$, $5.85(\mathrm{~s}, 1 \mathrm{H}), 2.32(\mathrm{~s}, 3 \mathrm{H}) ;{ }^{13} \mathrm{C} \mathrm{NMR}\left(101 \mathrm{MHz}, \mathrm{CDCl}_{3}\right) \delta$ : $143.97,136.64,128.70,128.19,127.04,123.57,121.89$, $119.91,119.66,119.19,111.01,109.99,40.18$. HRMS $\left(\mathrm{ESI}^{+}\right.$) calcd for $\mathrm{C}_{24} \mathrm{H}_{21} \mathrm{~N}_{2}[\mathrm{M}+\mathrm{H}]^{+}$337.1699, found 337.1698 .

3,3'-((4-Ethylphenyl)methylene)bis( $1 H$-indole) $\quad$ (3c): $92 \%$ yield, reddish brown solid, m.p. 82 84 ${ }^{\circ} \mathrm{C} ;{ }^{1} \mathrm{H}$ NMR $\left(400 \mathrm{MHz}, \mathrm{CDCl}_{3}\right) \delta: 7.72(\mathrm{~s}, 2 \mathrm{H}), 7.44(\mathrm{~d}, J=7.9 \mathrm{~Hz}$, 2H), $7.29(\mathrm{t}, J=7.6 \mathrm{~Hz}, 4 \mathrm{H}), 7.21 \sim 7.17(\mathrm{~m}, 2 \mathrm{H}), 7.14(\mathrm{~d}$, $J=8.1 \mathrm{~Hz}, 2 \mathrm{H}), 7.07 \sim 7.00(\mathrm{~m}, 2 \mathrm{H}), 6.58 \sim 6.57(\mathrm{~m}, 2 \mathrm{H})$, $5.88(\mathrm{~s}, 1 \mathrm{H}), 2.67(\mathrm{q}, J=7.6 \mathrm{~Hz}, 2 \mathrm{H}), 1.27(\mathrm{t}, J=7.6 \mathrm{~Hz}$, $3 \mathrm{H}) ;{ }^{13} \mathrm{C} \mathrm{NMR}\left(101 \mathrm{MHz}, \mathrm{CDCl}_{3}\right) \delta: 141.99,141.40$, $136.79,128.74,127.82,127.24,123.77,121.96,120.09$, 119.93, 119.27, 111.24, 39.90, 28.59, 15.67. HRMS (ESI $\left.{ }^{+}\right)$ calcd for $\mathrm{C}_{25} \mathrm{H}_{23} \mathrm{~N}_{2}[\mathrm{M}+\mathrm{H}]^{+}$351.1856, found 356.1854 .

3,3'-((4-(Tert-butyl)phenyl)methylene)bis(1H-indole) (3d): $89 \%$ yield, reddish brown solid, m.p. 97.1 99.1 ${ }^{\circ} \mathrm{C}$; ${ }^{1} \mathrm{H}$ NMR (400 MHz, $\left.\mathrm{CDCl}_{3}\right) \delta: 7.88(\mathrm{~s}, 2 \mathrm{H}), 7.40(\mathrm{~d}, J=8.0$ $\mathrm{Hz}, 2 \mathrm{H}), 7.34(\mathrm{dt}, J=8.2,0.8 \mathrm{~Hz}, 2 \mathrm{H}), 7.26(\mathrm{~m}$, 4H), $7.17 \sim 7.13(\mathrm{~m}, 2 \mathrm{H}), 6.99(\mathrm{ddd}, J=8.0,7.0,1.0 \mathrm{~Hz}$, $2 \mathrm{H}), 6.69 \sim 6.68(\mathrm{~m}, 2 \mathrm{H}), 5.85(\mathrm{~s}, 1 \mathrm{H}), 1.29(\mathrm{~s}, 9 \mathrm{H}) ;{ }^{13} \mathrm{C}$ NMR $\left(101 \mathrm{MHz} \mathrm{CDCl}_{3}\right){ }^{13} \mathrm{C}$ NMR $\delta: 148.79,140.88$, $136.77,128.30,127.24,125.11,123.60,121.93,120.10$, 119.21, 111.04, 100.00, 39.72, 34.44, 31.53. HRMS (ESI $\left.{ }^{+}\right)$ calcd for $\mathrm{C}_{27} \mathrm{H}_{27} \mathrm{~N}_{2}[\mathrm{M}+\mathrm{H}]^{+} 379.2169$, found 379.2171.

3,3'-((4-Bromophenyl)methylene)bis(1H-indole) $\quad(\mathbf{3 e})$ : $91 \%$ yield, reddish brown solid, m.p. $84.2 \sim 86.2{ }^{\circ} \mathrm{C} ;{ }^{1} \mathrm{H}$ NMR (400 MHz, $\left.\mathrm{CDCl}_{3}\right) \delta: 7.70(\mathrm{~s}, 2 \mathrm{H}), 7.43$ (dd, $J=7.8$, $4.3 \mathrm{~Hz}, 4 \mathrm{H}), 7.31(\mathrm{~d}, J=8.1 \mathrm{~Hz}, 2 \mathrm{H}), 7.27 \sim 7.20(\mathrm{~m}, 4 \mathrm{H})$, $7.12 \sim 7.03(\mathrm{~m}, 2 \mathrm{H}), 6.55 \sim 6.48(\mathrm{~m}, 2 \mathrm{H}), 5.88(\mathrm{~s}, 1 \mathrm{H}) ;{ }^{13} \mathrm{C}$ NMR (101 MHz, $\left.\mathrm{CDCl}_{3}\right) \delta: 143.32,136.81,131.51$, $130.69,127.02,123.90,122.25,120.10,119.97,119.54$, 119.07, 111.46, 39.84. HRMS (ESI ${ }^{+}$) calcd for $\mathrm{C}_{23} \mathrm{H}_{18} \mathrm{BrN}_{2}$ $[\mathrm{M}+\mathrm{H}]^{+}$401.0648, found 401.0650 .

3,3'-((4-Nitrophenyl)methylene)bis( $1 H$-indole) (3f): $90 \%$ yield, orange solid, m.p. $239 \sim 241{ }^{\circ} \mathrm{C} ;{ }^{1} \mathrm{H}$ NMR $(400$ $\left.\mathrm{MHz}, \mathrm{CDCl}_{3}\right) \delta: 8.13(\mathrm{~d}, J=8.8 \mathrm{~Hz}, 2 \mathrm{H}), 8.00(\mathrm{~s}, 2 \mathrm{H})$, 7.50 (d, $J=8.4 \mathrm{~Hz}, 2 \mathrm{H}), 7.38$ (d, $J=8.2 \mathrm{~Hz}, 2 \mathrm{H}), 7.33$ (d, $J=7.8 \mathrm{~Hz}, 2 \mathrm{H}), 7.19$ (ddd, $J=8.2,7.1,1.2 \mathrm{~Hz}, 2 \mathrm{H}), 7.02$ (ddd, $J=8.0,7.1,0.9 \mathrm{~Hz}, 2 \mathrm{H}), 6.68 \sim 6.67(\mathrm{~m}, 2 \mathrm{H}), 5.98$ 
$(\mathrm{s}, 1 \mathrm{H}) ;{ }^{13} \mathrm{C} \mathrm{NMR}\left(101 \mathrm{MHz}, \mathrm{CDCl}_{3}\right) \delta: 151.91,136.77$, $129.61,126.73,123.71,123.70,122.44,119.70,119.64$, 118.23, 111.34, 100.00, 40.29. HRMS (ESI ${ }^{+}$) calcd for $\mathrm{C}_{23} \mathrm{H}_{18} \mathrm{~N}_{3} \mathrm{O}_{2}[\mathrm{M}+\mathrm{H}]^{+}$368.1394, found 368.1393.

3,3'-((3-Methoxyphenyl)methylene)bis( $1 H$-indole) (3g): $92 \%$ yield, reddish brown solid; m.p. $175 \sim 179{ }^{\circ} \mathrm{C} ;{ }^{1} \mathrm{H}$ NMR (400 MHz, $\left.\mathrm{CDCl}_{3}\right) \delta: 7.88(\mathrm{~s}, 2 \mathrm{H}), 7.40(\mathrm{~d}, J=7.7$ $\mathrm{Hz}, 2 \mathrm{H}), 7.33(\mathrm{~d}, J=8.1 \mathrm{~Hz}, 2 \mathrm{H}), 7.21 \sim 7.14(\mathrm{~m}, 3 \mathrm{H})$, $7.02 \sim 6.98(\mathrm{~m}, 2 \mathrm{H}), 6.94(\mathrm{~d}, J=7.6 \mathrm{~Hz}, 1 \mathrm{H}), 6.75$ (ddd, $J=8.2,2.6,0.8 \mathrm{~Hz}, 1 \mathrm{H}), 6.92 \sim 6.91(\mathrm{~m}, 1 \mathrm{H}), 6.65 \sim 6.64$ $(\mathrm{m}, 2 \mathrm{H}), 5.85(\mathrm{~s}, 1 \mathrm{H}), 3.72(\mathrm{~s}, 3 \mathrm{H}) ;{ }^{13} \mathrm{C} \mathrm{NMR}(101 \mathrm{MHz}$, $\left.\mathrm{CDCl}_{3}\right) \delta: 159.64,145.83,136.76,129.19,127.17,123.66$, $121.98,121.38,119.98,119.63,119.30,114.83,111.34$, 111.09, 55.19, 40.31. HRMS $\left(\right.$ ESI $\left.^{+}\right)$calcd for $\mathrm{C}_{24} \mathrm{H}_{21} \mathrm{~N}_{2} \mathrm{O}$ $[\mathrm{M}+\mathrm{H}]^{+}$353.1649, found 353.1651.

3,3'-((3-Bromophenyl)methylene)bis( $1 H$-indole) (3h): $91 \%$ yield, reddish brown solid, m.p. $85 \sim 87{ }^{\circ} \mathrm{C} ;{ }^{1} \mathrm{H}$ NMR $\left(400 \mathrm{MHz} \mathrm{CDCl}_{3}\right) \delta: 7.62(\mathrm{~s}, 2 \mathrm{H}), 7.59(\mathrm{t}, J=1.5 \mathrm{~Hz}, 1 \mathrm{H})$, $7.47(\mathrm{~d}, J=7.9 \mathrm{~Hz}, 2 \mathrm{H}), 7.43(\mathrm{~d}, J=7.9 \mathrm{~Hz}, 1 \mathrm{H}), 7.32 \sim$ $7.24(\mathrm{~m}, 5 \mathrm{H}), 7.16(\mathrm{~d}, J=7.8 \mathrm{~Hz}, 1 \mathrm{H}), 7.14 \sim 7.10(\mathrm{~m}$, $2 \mathrm{H}), 6.52 \sim 6.47(\mathrm{~m}, 2 \mathrm{H}), 5.91(\mathrm{~s}, 1 \mathrm{H}) ;{ }^{13} \mathrm{C} \mathrm{NMR}(101$ $\left.\mathrm{MHz}, \mathrm{CDCl}_{3}\right) \delta: 146.80,136.82,131.88,130.15,129.61$, $127.63,127.03,124.01,122.65,122.30,119.95,119.60$, 118.90, 111.54, 40.13. HRMS (ESI ${ }^{+}$) calcd for $\mathrm{C}_{23} \mathrm{H}_{18} \mathrm{BrN}_{2}$ $[\mathrm{M}+\mathrm{H}]^{+}$401.0648, found 401.0646 .

3,3'-((3-(Trifluoromethyl)phenyl)methylene)bis(1H-indole) (3i): 90\% yield, reddish brown solid, m.p. 135 $137{ }^{\circ} \mathrm{C} ;{ }^{1} \mathrm{H}$ NMR (400 MHz, $\left.\mathrm{CDCl}_{3}\right) \delta: 7.76(\mathrm{~s}, 2 \mathrm{H}), 7.71$ (s, 1H), 7.53 (d, $J=7.8 \mathrm{~Hz}, 2 \mathrm{H}), 7.42$ (d, $J=7.9 \mathrm{~Hz}, 2 \mathrm{H})$, $7.38(\mathrm{t}, J=7.8 \mathrm{~Hz}, 1 \mathrm{H}), 7.34(\mathrm{~d}, J=8.2 \mathrm{~Hz}, 2 \mathrm{H}), 7.26 \sim$ $7.22(\mathrm{~m}, 2 \mathrm{H}), 7.08(\mathrm{td}, J=7.6,0.8 \mathrm{~Hz}, 2 \mathrm{H}), 6.58 \sim 6.52(\mathrm{~m}$, 2H), $5.99(\mathrm{~s}, 1 \mathrm{H}) ;{ }^{13} \mathrm{C}$ NMR $\left(101 \mathrm{MHz}, \mathrm{CDCl}_{3}\right) \delta: 145.20$, $136.83,132.19,130.79,130.47,128.86,126.95,125.62$, $125.58,123.87,122.29,119.83,119.56,118.88,111.39$, 40.22. HRMS $\left(\mathrm{ESI}^{+}\right)$calcd for $\mathrm{C}_{24} \mathrm{H}_{18} \mathrm{~F}_{3} \mathrm{~N}_{2}[\mathrm{M}+\mathrm{H}]^{+}$ 391.1417 , found 391.1415 .

3,3'-((3-Nitrophenyl)methylene)bis( $1 H$-indole $)$

(3j): $89 \%$ yield, reddish brown solid, m.p. $163 \sim 165{ }^{\circ} \mathrm{C} ;{ }^{1} \mathrm{H}$ NMR (400 MHz, $\left.\mathrm{CDCl}_{3}\right) \delta: 8.21(\mathrm{~m}, 1 \mathrm{H}), 8.07$ (ddd, $J=$ $8.2,2.3,1.1 \mathrm{~Hz}, 1 \mathrm{H}), 7.96(\mathrm{~s}, 2 \mathrm{H}), 7.68$ (d, $J=7.6 \mathrm{~Hz}, 1 \mathrm{H})$, $7.43(\mathrm{t}, J=7.9 \mathrm{~Hz}, 1 \mathrm{H}), 7.38 \sim 7.34(\mathrm{~m}, 4 \mathrm{H}), 7.22 \sim 7.15$ $(\mathrm{m}, 2 \mathrm{H}), 7.06 \sim 6.96(\mathrm{~m}, 2 \mathrm{H}), 6.65 \sim 6.65(\mathrm{~m}, 2 \mathrm{H}), 5.99(\mathrm{~s}$, $1 \mathrm{H}) ;{ }^{13} \mathrm{C} \mathrm{NMR}\left(101 \mathrm{MHz}, \mathrm{CDCl}_{3}\right) \delta: 148.57,146.46$, $136.82,134.97,129.22,126.72,123.77,123.70,122.41$, $121.59,119.65,119.63,118.37,111.37,40.10$. HRMS $\left(\mathrm{ESI}^{+}\right.$) calcd for $\mathrm{C}_{23} \mathrm{H}_{18} \mathrm{~N}_{3} \mathrm{O}_{2}[\mathrm{M}+\mathrm{H}]^{+}$368.1394; found 368.1392 .

3,3'-((2-Methoxyphenyl)methylene)bis( $1 H$-indole) (3k): $87 \%$ yield, reddish brown solid, m.p. 96 98 ${ }^{\circ} \mathrm{C} ;{ }^{1} \mathrm{H}$ NMR $\left(400 \mathrm{MHz}, \mathrm{CDCl}_{3}\right) \delta: 7.80(\mathrm{~s}, 2 \mathrm{H}), 7.40(\mathrm{~d}, J=7.8 \mathrm{~Hz}$, 2H), $7.31(\mathrm{~d}, J=8.1 \mathrm{~Hz}, 2 \mathrm{H}), 7.18(\mathrm{~s}, 1 \mathrm{H}), 7.14$ (t, $J=7.2$ $\mathrm{Hz}, 3 \mathrm{H}), 6.98$ (t, $J=7.5 \mathrm{~Hz}, 2 \mathrm{H}), 6.92(\mathrm{~d}, J=8.2 \mathrm{~Hz}, 1 \mathrm{H})$, $6.80(\mathrm{t}, J=7.5 \mathrm{~Hz}, 1 \mathrm{H}), 6.61(\mathrm{~s}, 2 \mathrm{H}), 6.34(\mathrm{~s}, 1 \mathrm{H}), 3.80(\mathrm{~s}$, $3 \mathrm{H}) ;{ }^{13} \mathrm{C} \mathrm{NMR}\left(101 \mathrm{MHz}, \mathrm{CDCl}_{3}\right) \delta: 156.86,136.67$, $132.32,129.69,127.22,123.49,123.45,121.72,120.36$, $120.00,119.56,118.99,110.90,110.57,31.97,28.24$. HRMS $\left(\mathrm{ESI}^{+}\right.$) calcd for $\mathrm{C}_{24} \mathrm{H}_{21} \mathrm{~N}_{2} \mathrm{O}[\mathrm{M}+\mathrm{H}]^{+} 353.1649$, found 353.1651 .

3,3'-((2-Chlorophenyl)methylene)bis( $1 H$-indole)

(31): $85 \%$ yield, reddish brown solid, m.p. 95 97 ${ }^{\circ} \mathrm{C} ;{ }^{1} \mathrm{H}$ NMR $\left(400 \mathrm{MHz}, \mathrm{CDCl}_{3}\right) \delta: 8.01(\mathrm{~d}, J=8.0 \mathrm{~Hz}, 1 \mathrm{H}), 7.86(\mathrm{~s}$, 2H), $7.48(\mathrm{t}, J=7.7 \mathrm{~Hz}, 1 \mathrm{H}), 7.43 \sim 7.36(\mathrm{~m}, 3 \mathrm{H}), 7.33(\mathrm{~d}$, $J=8.2 \mathrm{~Hz}, 2 \mathrm{H}), 7.19$ (d, $J=5.7 \mathrm{~Hz}, 1 \mathrm{H}), 7.15$ (d, $J=8.0$ $\mathrm{Hz}, 2 \mathrm{H}), 7.08$ (t, $J=7.4 \mathrm{~Hz}, 1 \mathrm{H}), 7.01(\mathrm{t}, J=7.5 \mathrm{~Hz}, 2 \mathrm{H})$, $6.59(\mathrm{~s}, 1 \mathrm{H}), 6.33(\mathrm{~s}, 1 \mathrm{H}) ;{ }^{13} \mathrm{C} \mathrm{NMR}\left(101 \mathrm{MHz}, \mathrm{CDCl}_{3}\right) \delta$ : $170.08,141.26,136.67,132.42,127.48,126.95,126.61$, 123.77, 123.73, 121.99, 119.82, 119.28, 118.31, 111.04, 36.57. HRMS $\left(\mathrm{ESI}^{+}\right.$) calcd for $\mathrm{C}_{23} \mathrm{H}_{18} \mathrm{ClN}_{2}[\mathrm{M}+\mathrm{H}]^{+}$ 357.1153 , found 357.1151 .

3,3'-((2,4-Dimethylphenyl)methylene $)$ bis( $1 H$-indole $)$ (3m): 83\% yield, reddish brown solid, m.p. $180 \sim 182{ }^{\circ} \mathrm{C}$; ${ }^{1} \mathrm{H}$ NMR (400 MHz, $\left.\mathrm{CDCl}_{3}\right) \delta: 7.81(\mathrm{~s}, 2 \mathrm{H}), 7.38 \sim 7.32$ $(\mathrm{m}, 4 \mathrm{H}), 7.19 \sim 7.14(\mathrm{~m}, 2 \mathrm{H}), 7.04 \sim 6.96(\mathrm{~m}, 4 \mathrm{H}), 6.84(\mathrm{~d}$, $J=7.3 \mathrm{~Hz}, 1 \mathrm{H}), 6.56 \sim 6.53(\mathrm{~m}, 2 \mathrm{H}), 5.98(\mathrm{~s}, 1 \mathrm{H}), 2.35(\mathrm{~s}$, $3 \mathrm{H}), 2.30(\mathrm{~s}, 3 \mathrm{H}) ;{ }^{13} \mathrm{C} \mathrm{NMR}\left(101 \mathrm{MHz}, \mathrm{CDCl}_{3}\right) \delta: 139.12$, $136.82,135.87,135.43,131.11,128.43,127.30,126.56$, $123.89,121.94,119.91,119.45,119.24,111.09,35.96$, 21.07, 19.55. HRMS (ESI $\left.{ }^{+}\right)$calcd for $\mathrm{C}_{25} \mathrm{H}_{23} \mathrm{~N}_{2}[\mathrm{M}+\mathrm{H}]^{+}$ 351.1856 , found 351.1858 .

3,3'-((2,6-Dimethylphenyl)methylene)bis( $1 H$-indole) (3n): $77 \%$ yield, reddish brown solid, m.p. $172 \sim 174{ }^{\circ} \mathrm{C}$; ${ }^{1} \mathrm{H}$ NMR $\left(400 \mathrm{MHz}, \mathrm{CDCl}_{3}\right) \delta: 7.82(\mathrm{~s}, 2 \mathrm{H}), 7.35(\mathrm{t}, J=7.8$ $\mathrm{Hz}, 4 \mathrm{H}), 7.18 \sim 7.14(\mathrm{~m}, 2 \mathrm{H}), 7.05 \sim 6.98(\mathrm{~m}, 3 \mathrm{H}), 6.96 \sim$ $6.90(\mathrm{~m}, 2 \mathrm{H}), 6.54 \sim 6.54(\mathrm{~m}, 2 \mathrm{H}), 6.08(\mathrm{~s}, 1 \mathrm{H}), 2.33(\mathrm{~s}$, $3 \mathrm{H}), 2.27(\mathrm{~s}, 3 \mathrm{H}) ;{ }^{13} \mathrm{C}$ NMR $\left(101 \mathrm{MHz}, \mathrm{CDCl}_{3}\right) \delta 141.99$, $136.80,136.54,134.67,127.97,127.29,126.45,125.17$, $124.03,121.94,119.91,119.61,119.26,111.09,36.61$, 21.04, 15.09. HRMS $\left(\mathrm{ESI}^{+}\right)$calcd for $\mathrm{C}_{25} \mathrm{H}_{23} \mathrm{~N}_{2}[\mathrm{M}+\mathrm{H}]^{+}$ 351.1856 , found 351.1858 .

3,3'-((2,3-Dimethylphenyl)methylene)bis( $1 H$-indole $)$ (3o): $86 \%$ yield, reddish brown solid, m.p. $248 \sim 250{ }^{\circ} \mathrm{C}$; ${ }^{1} \mathrm{H}$ NMR (400 MHz, $\left.\mathrm{CDCl}_{3}\right) \delta: 7.86(\mathrm{~s}, 2 \mathrm{H}), 7.36(\mathrm{dt}, J=$ $8.1,0.8 \mathrm{~Hz}, 2 \mathrm{H}), 7.29 \sim 7.26(\mathrm{~m}, 2 \mathrm{H}), 7.17$ (ddd, $J=8.2$, $7.1,1.1 \mathrm{~Hz}, 2 \mathrm{H}), 7.11 \sim 7.07(\mathrm{~m}, 1 \mathrm{H}), 7.02 \sim 6.97(\mathrm{~m}, 4 \mathrm{H})$, $6.64(\mathrm{dd}, J=2.4,1.3 \mathrm{~Hz}, 2 \mathrm{H}), 6.26(\mathrm{t}, J=1.2 \mathrm{~Hz}, 1 \mathrm{H}), 2.18$ $(\mathrm{s}, 3 \mathrm{H}), 1.26(\mathrm{~s}, 3 \mathrm{H}) ;{ }^{13} \mathrm{C} \mathrm{NMR}\left(101 \mathrm{MHz}, \mathrm{CDCl}_{3}\right) \delta$ : $141.99,136.80,136.54,134.67,127.97,127.29,126.45$, $125.17,124.03,121.94,119.91,119.61,119.26,111.09$, 36.61, 21.04, 15.09. HRMS $\left(\mathrm{ESI}^{+}\right)$calcd for $\mathrm{C}_{25} \mathrm{H}_{23} \mathrm{~N}_{2}$ $[\mathrm{M}+\mathrm{H}]^{+}$351.1856, found 351.1858.

3,3'-((3,4-Dimethoxyphenyl)methylene)bis( $1 H$-indole $)$ (3p): 91\% yield, reddish brown solid, m.p. $186 \sim 188{ }^{\circ} \mathrm{C}$; ${ }^{1} \mathrm{H}$ NMR $\left(400 \mathrm{MHz}, \mathrm{CDCl}_{3}\right) \delta: 7.90(\mathrm{~s}, 2 \mathrm{H}), 7.39(\mathrm{~d}, J=$ $8.0 \mathrm{~Hz}, 2 \mathrm{H}), 7.34(\mathrm{~d}, J=8.2 \mathrm{~Hz}, 2 \mathrm{H}), 7.18 \sim 7.14(\mathrm{~m}, 2 \mathrm{H})$, 7.00 (ddd, $J=8.0,7.1,0.9 \mathrm{~Hz}, 2 \mathrm{H}), 6.92(\mathrm{~m}, 1 \mathrm{H}), 6.83$ (dd, $J=8.2,1.9 \mathrm{~Hz}, 1 \mathrm{H}), 6.76(\mathrm{~d}, J=8.3 \mathrm{~Hz}, 1 \mathrm{H}), 6.63(\mathrm{~m}$, $2 \mathrm{H}), 5.83(\mathrm{~s}, 1 \mathrm{H}), 3.84(\mathrm{~s}, 3 \mathrm{H}), 3.75(\mathrm{~s}, 3 \mathrm{H}) ;{ }^{13} \mathrm{C} \mathrm{NMR}$ $\left(101 \mathrm{MHz}, \mathrm{CDCl}_{3}\right) \delta: 148.77,147.39,136.82,136.79$, $127.16,123.63,122.00,120.67,120.04,119.98,119.97$, $119.29,112.32,111.12,110.99,55.92,39.89$. HRMS $\left(\mathrm{ESI}^{+}\right.$) calcd for $\mathrm{C}_{25} \mathrm{H}_{23} \mathrm{~N}_{2} \mathrm{O}_{2}[\mathrm{M}+\mathrm{H}]^{+}$383.1754, found 383.1752 .

3,3'-(Phenylmethylene)bis(2-methyl-1 $H$-indole) $\quad$ (3q): $96 \%$ yield, reddish brown solid, m.p. $234 \sim 236{ }^{\circ} \mathrm{C} ;{ }^{1} \mathrm{H}$ 
NMR (400 MHz, $\left.\mathrm{CDCl}_{3}\right) \delta: 7.73(\mathrm{~s}, 2 \mathrm{H}), 7.24(\mathrm{~d}, J=10.5$ $\mathrm{Hz}, 7 \mathrm{H}), 7.03$ (t, $J=7.5 \mathrm{~Hz}, 2 \mathrm{H}), 6.97$ (d, $J=7.9 \mathrm{~Hz}, 2 \mathrm{H})$, $6.85(\mathrm{t}, J=7.5 \mathrm{~Hz}, 2 \mathrm{H}), 6.00(\mathrm{~s}, 1 \mathrm{H}), 2.06(\mathrm{~s}, 6 \mathrm{H}) ;{ }^{13} \mathrm{C}$ NMR $\left(101 \mathrm{MHz}, \mathrm{CDCl}_{3}\right) \delta: 135.00,131.75,129.05$, $128.95,128.06,128.03,120.57,119.30,119.03,117.99$, 113.38, 109.90, 39.22, 12.42. HRMS $\left(\mathrm{ESI}^{+}\right)$calcd for $\mathrm{C}_{25} \mathrm{H}_{23} \mathrm{~N}_{2}[\mathrm{M}+\mathrm{H}]^{+}$351.1856, found 351.1854.

3,3'-(Phenylmethylene)bis(5-bromo- $1 H$-indole) (3r): $94 \%$ yield, reddish brown solid, m.p. $235 \sim 237{ }^{\circ} \mathrm{C} ;{ }^{1} \mathrm{H}$ NMR (400 MHz, $\left.\mathrm{CDCl}_{3}\right) \delta: 7.97(\mathrm{~s}, 2 \mathrm{H}), 7.45(\mathrm{~s}, 2 \mathrm{H})$, $7.34 \sim 7.17(\mathrm{~m}, 9 \mathrm{H}), 6.63(\mathrm{~s}, 2 \mathrm{H}), 5.73(\mathrm{~s}, 1 \mathrm{H}) ;{ }^{13} \mathrm{C} \mathrm{NMR}$ $\left(101 \mathrm{MHz}, \mathrm{CDCl}_{3}\right) \delta: 143.01,135.30,128.64,128.51$, $128.41,126.51,124.95,122.26,119.05,117.99,112.66$, 112.55, 39.87. HRMS (ESI ${ }^{+}$) calcd for $\mathrm{C}_{23} \mathrm{H}_{17} \mathrm{Br}_{2} \mathrm{~N}_{2}[\mathrm{M}+$ $\mathrm{H}]^{+}$478.9753, found 478.9755 .

3,3'-(Ethane-1,1-diyl)bis(1H-indole) (3s): 91\% yield, light brown solid, m.p. 87 $89{ }^{\circ} \mathrm{C}$; ${ }^{1} \mathrm{H}$ NMR $(500 \mathrm{MHz}$, $\left.\mathrm{CDCl}_{3}\right) \delta: 7.71(\mathrm{~s}, 1 \mathrm{H}), 7.37(\mathrm{~d}, J=7.4 \mathrm{~Hz}, 1 \mathrm{H}), 7.29(\mathrm{t}$, $J=7.7 \mathrm{~Hz}, 2 \mathrm{H}), 7.22(\mathrm{dd}, J=6.3,4.4 \mathrm{~Hz}, 1 \mathrm{H}), 7.17$ (d, $J=$ $7.0 \mathrm{~Hz}, 1 \mathrm{H}), 7.11(\mathrm{t}, J=7.6 \mathrm{~Hz}, 1 \mathrm{H}), 6.91(\mathrm{t}, J=8.0 \mathrm{~Hz}$, $1 \mathrm{H}), 6.53(\mathrm{~d}, J=2.3 \mathrm{~Hz}, 1 \mathrm{H}), 2.33(\mathrm{~s}, 2 \mathrm{H}) ;{ }^{13} \mathrm{C}$ NMR $(126$ $\left.\mathrm{MHz}, \mathrm{CDCl}_{3}\right) \delta: 136.66,126.93,121.80,121.68,121.25$, 119.76, 119.04, 111.11, 28.20, 21.77. HRMS (ESI $\left.{ }^{+}\right)$ calcd for $\mathrm{C}_{18} \mathrm{H}_{17} \mathrm{~N}_{2}[\mathrm{M}+\mathrm{H}]^{+}$261.1373, found 261.1371.

3,3'-(1-Phenylethane-1,1-diyl)bis(1H-indole) (3s): $86 \%$ yield, light blue solid, m.p. 94 $96{ }^{\circ} \mathrm{C} ;{ }^{1} \mathrm{H}$ NMR $(500$ $\left.\mathrm{MHz} \mathrm{CDCl}_{3}\right) \delta: 7.71(\mathrm{~s}, 1 \mathrm{H}), 7.37(\mathrm{~d}, J=7.4 \mathrm{~Hz}, 1 \mathrm{H})$, $7.29(\mathrm{t}, J=7.7 \mathrm{~Hz}, 2 \mathrm{H}), 7.24 \sim 7.20(\mathrm{~m}, 1 \mathrm{H}), 7.17(\mathrm{~d}, J=$ $7.0 \mathrm{~Hz}, 1 \mathrm{H}), 7.11(\mathrm{t}, J=7.6 \mathrm{~Hz}, 1 \mathrm{H}), 6.91(\mathrm{t}, J=8.0 \mathrm{~Hz}$, $1 \mathrm{H}), 6.53(\mathrm{~d}, J=2.3 \mathrm{~Hz}, 1 \mathrm{H}), 2.33(\mathrm{~s}, 2 \mathrm{H}) ;{ }^{13} \mathrm{C}$ NMR $(126$ $\left.\mathrm{MHz}, \mathrm{CDCl}_{3}\right) \delta: 148.04,137.10,128.10,127.82,126.47$, $125.84,124.71,123.42,122.11,121.55,118.94,111.20$, 43.76, 28.79. HRMS (ESI ${ }^{+}$) calcd for $\mathrm{C}_{24} \mathrm{H}_{21} \mathrm{~N}_{2}[\mathrm{M}+\mathrm{H}]^{+}$ 336.1673 , found 336.1677 .

Supporting Information Detailed experimental procedures and hydrogen and carbon spectrum data. The Supporting Information is available free of charge via the Internet at http://sioc-journal.cn/.

\section{References}

[1] (a) Garbe, T. R.; Kobayashi, M.; Shimizu, N.; Takesue, N.; Ozawa, M.; Yukawa, H. J. Nat. Prod. 2000, 63, 596.

(b) Bifulco, G.; Bruno, I.; Riccio, R.; Lavayre, J.; Bourdy, G. J. Nat. Prod. 1995, 58, 1254.

[2] (a) Shiri, M.; Zolfigol, M. A.; Kruger, H. G.; Tanbakouchian, Z. Chem Rev. 2010, 110, 2250.

(b) Bell, R.; Carmeli, S.; Sar, N. J. Nat. Prod. 1994, 57, 1587.

(c) Fahy, E.; Potts, B. C. M.; Faulkner, D. J.; Smith, K. J. Nat. Prod. 1991, 54, 564.

(d) Swetha, A.; MadhuBabu, B.; Meshram. H. M. Tetrahedron Lett. 2015, 56, 1775 .

[3] Bharate, S. B.; Bharate, J. B.; Khan, S. I.; Tekwani, B. L.; Jacob, M. R.; Mudududdla, R.; Yadav, R. R.; Singh, B. ; Sharma, P. R.; Maity, S.; Singh, B.; Khan, I. A.; Vishwakarma, R. A. Eur. J. Med. Chem. 2013, 63, 435 .

[4] Sarva, S.; Harinath, J. S.; Sthanikam, S. P.; Ethiraj, S.; Vaithiyalingam, M.; Cirandur, S. R. Chin. Chem. Lett. 2016, 27, 16.

[5] (a) Sashidhara, K. V.; Kumar, A.; Kumar, M.; Srivastava, A.; Puri,
A. Bioorg. Med. Chem. Lett. 2010, 20, 6504.

(b) Sashidhara, K. V.; Modukuri,R. K.; Sonkar, R.; Rao, K. B.; Bhatia, G. Eur. J. Med. Chem., 2013, 68, 38.

(c) Sashidhara, K. V.; Kumar, A.; Kumar, M.; Srivastava, A.; Puri, A. Bioorg. Med. Chem. Lett. 2010, 20, 6504.

[6] Lo, K. K. W.; Tsang, K. H. K.; Hui, W. K.; Zhu, N. Chem. Commun. 2003, 2704.

[7] (a) Mahboobi, S.; Uecker, A.; Sellmer, A.; Cénac, C.; Höcher, H.; Pongratz, H.; Eichhorn, E.; Hufsky, H.; Trümpler, A.; Sicker, M.; Heidel, F.; Fischer, T.; Stocking, C.; Elz, S.; Böhmer, F. D.; Dove, S. J. Med. Chem. 2006, 49, 3101.

(b) Kamal, A.; Srikanth, Y. V. V.; Ramaiah, M. J.; Khan, M. N. A.; Reddy, M. K.; Ashraf, M.; Lavanya, A.; Pushpavalli, S. N. C. V. L.; Pal-Bhadra, M. Bioorg. Med. Chem. Lett. 2012, 22, 571 .

(c) Safe, S.; Papineni, S.; Chintharlapalli, S. Cancer Lett. 2008, 26, 326.

(d) Grosso, C.; Cardoso, A. L.; Lemos, A.; Varela, J.; Rodrigues, M. J.; Custodio, L. L.; Barreira, T. M. Eur. J. Med. Chem. 2015, 93, 9-15.

(e) Rahimi, M.; Huang, K.-L.; Tang, C. K. Cancer Lett. 2010, 295, 59.

(f) Li, Y. W.; VandenBoom, T. G.; Wang, Z. W.; Kong, D. J.; Ali, S.; Philip, P. A.; Sarkar, F. H. Cancer Res. 2010, 70, 1486.

(g) Dawson, M. I.; Ye, M.; Cao, X. H.; Farhana, L; Hu, Q. Y.; Zhao, Y.; Xu, L. P.; Kiselyuk, A.; Correa, R. G.; Yang, L.; Hou, T. J.; Reed, J. C.; Itkin-Ansari, P.; Levine, F., Sanner, M. F.; Fontana, J. A.; Zhang. X.-K. ChemMedChem 2009, 4, 1106.

[8] Plimmer, J. R.; Gammon, D. W.; Ragsdale, N. N. Encyclopedia of Agrochemicals, John Wiley and Sons, New York, 2003, 3.

[9] (a) Cherioux, F.; Guyard, L.; Audebert, P. Adv. Mater. 1998, 10, 1013.

(b) Noack, A.; Schr€oder, A.; Hartmann, H. Dyes Pigm. 2002, 57, 131 .

[10] (a) Minne, S. C.; Manalis, S. R.; Quate, C. F. Appl. Phys. Lett. 1995, 67, 3918.

(b) Seyedi, N.; Khabazzadeh, H. Res. Chem. Intermed. 2015, 41, 2603.

(c) Praveen, P. J.; Parameswaran, P. S.; Majik, M. S. Synthesis 2015, 47, 1827.

(d) Shiri, M.; Zolfigol, M. A.; Kruger, H. G.; Tanbakouchian, Z.; Chem. Rev. 2010, 110, 2250.

[11] (a) Noland, W. E.; Kumar, H. V.; Flick, G. C.; Aspros, C. L.; Yoon, J. H.; Wilt, A. C.; Dehkordi, N.; Thao, S.; Schneerer, A. K.; Gao, S. M.; Tritch. K. J. Tetrahedron 2017, 73, 3913.

(b) Veisi, H.; Maleki, B.; Eshbala, F. H.; Veisi, H.; Masti, R.; Ashrafi, S. S.; Baghayeri, M. RSC Adv. 2014, 4, 30683.

[12] Chhattise, P. K.; Arbuj, S. S.; Mohite, K. C.; Bhavsar, S. V.; Horne, A. S.; Handore, K. N.; Chabukswar, V. V. RSC Adv. 2014, 4, 28623.

[13] Ravia, K.; Krishnakumara, B.; Swaminathana, M. Synth. React. Inorg. Met.-Org. Nano-Met. Chem. 2015, 45, 1380.

[14] (a) Mendes, S. R.; Thurow, S.; Penteado, F.; Silva, M. S. D.; Gariani, R. A.; Perin, G.; Lenardão, E. J. Green Chem. 2015, 17, 4334. (b) Ghorbani-Vaghei, R.; Veisi, H.; Keypour, H.; DehghaniFirouzabadi, A. A. Mol. Diversity 2010, 14, 87.

[15] Shirini, F.; Khaligh, N. G. Chin. J. Catal. 2013. 34, 1890.

[16] Tumtin, S.; Kathing, C.; Phucho, I. T.; Nongrum, R.; Myrboh, B.; Nongkhlaw, R. J. Chin. Chem. Soc. 2015, 62, 321.

[17] Veisi, H.; Gholbedaghi, R.; Malakootikhah, J.; Sedrpoushan, A.; Maleki, B.; Kordestani, D. J. Heterocycl. Chem. 2010, 47, 1398.

[18] Silveira, C. C.; Mendes, S. R.; Villetti, M. A.; Back, D. F.; Kaufman, T. S. Green Chem. 2012, 14, 2912.

[19] Liang, D. Q.; Huang, W. Z.; Yuan, L.; Ma, Y. H.; Ma, J. M.; Ning, D. M. Catal. Commun. 2014, 55, 11.

[20] Jejurkar, V. P.; Khatri, C. K.; Chaturbhuj, G. U.; Saha, S. ChemistrySelect 2017, 2, 11693.

[21] (a) Feng, X. L.; Guan, C. J.; Zhao, C. X. Synth. Commun. 2004, 34, 487.

(b) Surasania, R.; Kalitaa, D.; Chandrasekharb, K. B. Green Chem. 
Lett. Rev. 2013, 6, 113.

(c) Lin, Z. H.; Guan, C. J.; Feng, X. L.; Zhao, C. X. J. Mol. Catal. A: Chem. 2006, 247, 19.

[22] Fekri, L. Z.; Nikpassand, M.; Kohansal, M. Russ. J. Gen. Chem. 2015, 85, 2861 .

[23] Griffiths, K.; Kumar, P.; Akien, G. R.; Chilton, N. F.; Abdul-Sada, A.; Tizzard, G. J.; Coles, S. J.; Kostakis, G. E. Chem. Commun. 2016, 52,7866 .

[24] (a) Mobaraki, A.; Movassagh, B.;Karimi, B. Appl. Catal. A: Gen. 2014, 472, 123.

(b) Wang, A. Q.; Liu, X.; Su, Z. X.; Jing, H. W. Sci. Technol. 2014, 4, 71 .

(c) Rafiee, E.; Eavani, S.; Malaekeh-Nikouei, B. Chem. Lett. 2012, 41,438 .

[25] (a) Saffar-Teluri, A. Res. Chem. Intermed. 2014, 40, 1061.

(b) Shirini, F.; Lati, M. P. J. Iran. Chem. Soc. 2017, 14, 75.

(c) Sadeghi, B.; Tavasoli, F. A.; Hassanabadi, A. Synth. React. Inorg. Met.-Org. Nano-Met. Chem. 2015, 45, 1396.

[26] Shi, X. L.; Lin, H. K.; Li, P. Y.; Zhang, W. Q. ChemCatChem 2014, 6, 2947.

[27] Singh, N. G.; Nongrum, R.; Kathing, C.; Rani, J. W. S.; Nongkhlaw,
R. Green Chem. Lett. Rev. 2014, 7, 137.

[28] Gao, G.; Han, Y.; Zhang, Z. H. ChemistrySelect 2017, 2, 11561.

[29] Hirashita, T.; Ogawa, M.; Hattori, R.; Okochi, S.; Araki, S. Bull. Chem. Soc. Jpn. 2015, 88, 1760.

[30] (a) Wu, C.; Lu, L. H.; Peng, A. Z.; Jia, G. K.; Peng, C.; Cao, Z.; Tang, Z. L.; He, W. M.; Xu, X. H. Green Chem., 2018, 20, 3683.

(b) Zou, B. Yu, B.; Hu, C. W. J. $\mathrm{CO}_{2}$ Util. 2018, 26, 314.

(c) Xie, L. Y.; Peng, S.; Lu, L. H.; Hu, J.; Bao, W. H.; Zeng, F.; Tang, Z. L.; Xu, X. H.; He, W. M. ACS Sustainable Chem. Eng. 2018, 6, 7989.

[31] (a) Zhou, Z.; Duan, J. F.; Mu, X. J.; Xiao. S. Y. Chin. J. Org. Chem. 2018, 38, 585.

(b) Yue, H. L.; Bao, P. L.; Wang, L. L.; Lv, X. X.; Yang, D. S.; Wang, H.; Wei, W. Chin. J. Org. Chem. 2019, 39, 463.

(c) Zhao, S. Y.; Wang, Z. L. Chin. J. Org. Chem. 2016, 36, 862 (in Chinese).

(赵苏艳, 王祖利, 有机化学, 2016, 36, 862.)

(d) Li, W. Y.; Yin, G. X.; Huang, L.; Xiao, Y.; Fu, Z. M.; Xin, X.; Liu, F.; Li, Z. Z.; He, W. M. Green Chem. 2016, 18, 4879.

(e) Wu, C.; Xin, X.; Fu, Z. M.; Xie, L. Y.; Liu, K. J.; Wang, Z.; Li, W. Y.; Yuan, Z. H.; He, W. M. Green Chem. 2017, 19, 1983.

(Zhao, X.) 